\title{
WHOQOL-BREF survey of quality of life among dialyzed end-stage renal disease patients
}

Leszek Sułkowski ${ }^{1}$, Maciej Matyja ${ }^{2}$, Artur Pasternak ${ }^{3}$, Andrzej Matyja ${ }^{4}$

\begin{abstract}
${ }^{1}$ Department of General and Vascular Surgery, Regional Specialist Hospital, Częstochowa, Poland

${ }^{2} 2^{\text {nd }}$ Department of General Surgery, Department of Endoscopic, Metabolic and Soft Tissue Tumors Surgery, Jagiellonian University Medical College, Krakow, Poland ${ }^{3}$ Department of Anatomy, Jagiellonian University Medical College, Krakow, Poland ${ }^{4} 1^{\text {st }}$ Department of General, Oncological and Gastrointestinal Surgery, Jagiellonian University Medical College, Krakow, Poland
\end{abstract}

Submitted: 11 May 2018

Accepted: 28 September 2018

Arch Med Sci Civil Dis 2018; 3: e112-e120

DOI: https://doi.org/10.5114/amscd.2018.81057

Copyright $\odot 2018$ Termedia \& Banach

\section{Abstract}

Introduction: Hemodialysis (HD) improves health and prolongs the life of end-stage renal disease patients, but simultaneously leads to emotional disturbances and impairs the quality of life $(\mathrm{QOL})$. The study was conducted to evaluate the QOL of HD patients. The study was approved by the Regional Bioethical Committee (K.B.Cz.-0014/2017).

Material and methods: The World Health Organization Questionnaire of QOL (WHOQOL-BREF) was used in this study with the formal agreement of the WHO. The associations between each patient-related and dialysis-related factor and WHOQOL-BREF domains and questions were computed. The variables were compared by the Student $t$-test.

Results: Data were collected in August 2017 in a single access center. Sixty-nine patients, including 23 (33.3\%) women, were evaluated. The factors lowering the scores for particular questions and domains of WHOQOL-BREF were senility, marriage, wrist and arm AVF, not-tunneled CVCs (vs. tunneled), and unwillingness to have a kidney graft. The factors that increased scores for particular questions and domains of WHOQOL-BREF were short dialysis, tunneled CVCs (vs. not-tunneled), and higher URR. The relations between domains and questions of WHOQOL-BREF and sex, education, months on dialysis, kidney graft in the past, fulfillment of medical recommendations, $\mathrm{Kt} / \mathrm{V}$ and UF were not significant.

Conclusions: Although a kidney graft is the best kidney replacement therapy, there is a large group of patients who do not want to receive this treatment. This group should be given special attention. The medical professionals in HD units should remember that patients may not feel comfortable with their disease and satisfied with their body image affected by therapy.

Key words: quality of life, World Health Organization Questionnaire of Quality of Life, hemodialysis, end-stage renal disease, chronic kidney disease, vascular access.

\section{Introduction}

The growing population of end-stage renal disease (ESRD) patients require hemodialysis (HD), which is the most common renal replacement therapy. The HD improves health and prolongs life, but simultaneously

\author{
Corresponding author: \\ Leszek Sutkowski PhD \\ Department \\ of General and \\ Vascular Surgery \\ Regional Specialist \\ Hospital \\ 104/118 Bialska St \\ 42-218 Częstochowa, Poland \\ Phone: +48792 244177 \\ E-mail: leszeksulkowski@ \\ icloud.com
}


leads to emotional disturbances and reflects the quality of life (QOL) [1, 2]. All patients with chronic illnesses are reported to have impaired QOL [3], but ESRD patients on HD have considerably poorer QOL compared to healthy individuals, the general population and even to patients with other chronic diseases and to renal transplant patients [4-6]. The QOL is an important indicator of effective HD, a measure of patient outcome and an important variable assessing HD patients [1, 4, 7-9].

Due to the lack of measures assessing QOL, the World Health Organization (WHO) has developed its own instrument named the World Health Organization Questionnaire of Quality of Life (WHOQOL). The long version is the WHOQOL-100 containing 6 domains and 100 questions. The abbreviated questionnaire, WHOQOL-BREF, consists of 26 questions. According to the $\mathrm{WHO}$ definition, health is "a state of physical, mental and social well-being, not merely the absence of disease and infirmity". Thus both WHOQOL-100 and WHOQOL-BREF are focused on self-perceived QOL [10-13]. These questionnaires can be applied in broad-ranging ways, irrespectively of diseases, disabilities and symptoms [10-12, 14].

The aim of this study was to evaluate the QOL of ESRD patients undergoing HD with the WHOQOL-BREF questionnaire. The inclusion criteria were ESRD and $H D$, regardless of HD modality. The only exclusion criterion was lack of consent for participation. The patients received questionnaires at the dialysis session and were asked to complete them on the following day. The questionnaires were collected at the succeeding dialysis by medical staff.

The study was approved by the Bioethical Committee of the Regional Chambers of Physicians and Dentists (K.B.Cz.-0014/2017) and conducted in the Department of General and Vascular Surgery and the Dialysis Unit in the Regional Specialist Hospital in Częstochowa, Poland.

\section{Material and methods}

Data were collected in August 2017. At that time 135 patients were dialyzed in the Dialysis Unit in the Regional Specialist Hospital in Częstochowa, Poland. Each patient received information on a survey and was invited to participate. They received a questionnaire including demographic questions (age, gender, education, marital status), followed by medical history data (months on HD, modality of vascular access, kidney grafts, fulfillment of medical recommendations). Sixty-nine patients agreed to participate, gave formal consent in accordance with the requirements of the Bioethical Committee and were enrolled for further evaluation. Received data were supplemented with clinical information including $\mathrm{Kt} / \mathrm{V}$, urea reduction ratio (URR), ultrafiltration (UF), and duration of a single HD (Tables I-III). Finally, participants completed WHOQOL-BREF questionnaires. The association between each factor and each WHOQOL-BREF domain and separate question was computed and presented in Tables I-III.

Upon the formal agreement of the $\mathrm{WHO}$, the WHOQOL-BREF questionnaire was chosen for the study. The WHO provided the WHOQOL-BREF instrument along with a Code book. WHOQOL-BREF includes four domains (Physical health, Psychological, Social relationships and Environment, consisting of 7, 6, 3 and 8 questions, respectively) along with two separately scored questions about overall self-perception of QOL (question 1) and overall self-perception of health (question 2) [10-12]. The Physical health domain assesses activities of daily living, dependence on medicinal substances and medical aids, energy and fatigue, mobility, pain and discomfort, work capacity, sleep and rest. The Psychological domain covers: bodily image and appearance, negative and positive feelings, self-esteem, spirituality, religion and personal beliefs, thinking, learning, memory and concentration. The Social relationship domain includes: personal relationships, social support and sexual activity. The Environment domain assesses: financial resources; freedom, physical safety and security; accessibility and quality of health care and social care; home environment; opportunities for acquiring new information and skills; physical environment; participation in and opportunities for recreation and leisure activities [11, 12]. If in any of these domains more than $20 \%$ or at least 3 answers are missing, the whole domain assessment needs to be discarded. The Social relationship domain is an exception. Here only 1 missing answer is permissible. If one or two questions in a single domain are not answered, they may be substituted by the mean of the others in the same domain. In each domain the score is scaled positively, which means that a higher score corresponds to a higher QOL. Mean raw scores were transformed to a 0-100 scale according to the scoring procedures by multiplying by four $[11,12,15]$.

The factors which may affect the WHOQOLBREF score were analyzed. The demographic variables were: age, gender, marital status and education (Table I). The patient-related variables were: months on HD, vascular access, fulfillment of medical recommendations, kidney graft in the past or a desire to be a kidney graft recipient in the future (Table II). The dialysis-related variables were: duration of a single HD, Kt/V, URR and UF (Table III). The significance of association between each demographic, patient-related and dialysis-related factor and WHOQOL-BREF domains and two independent questions was evaluated. 


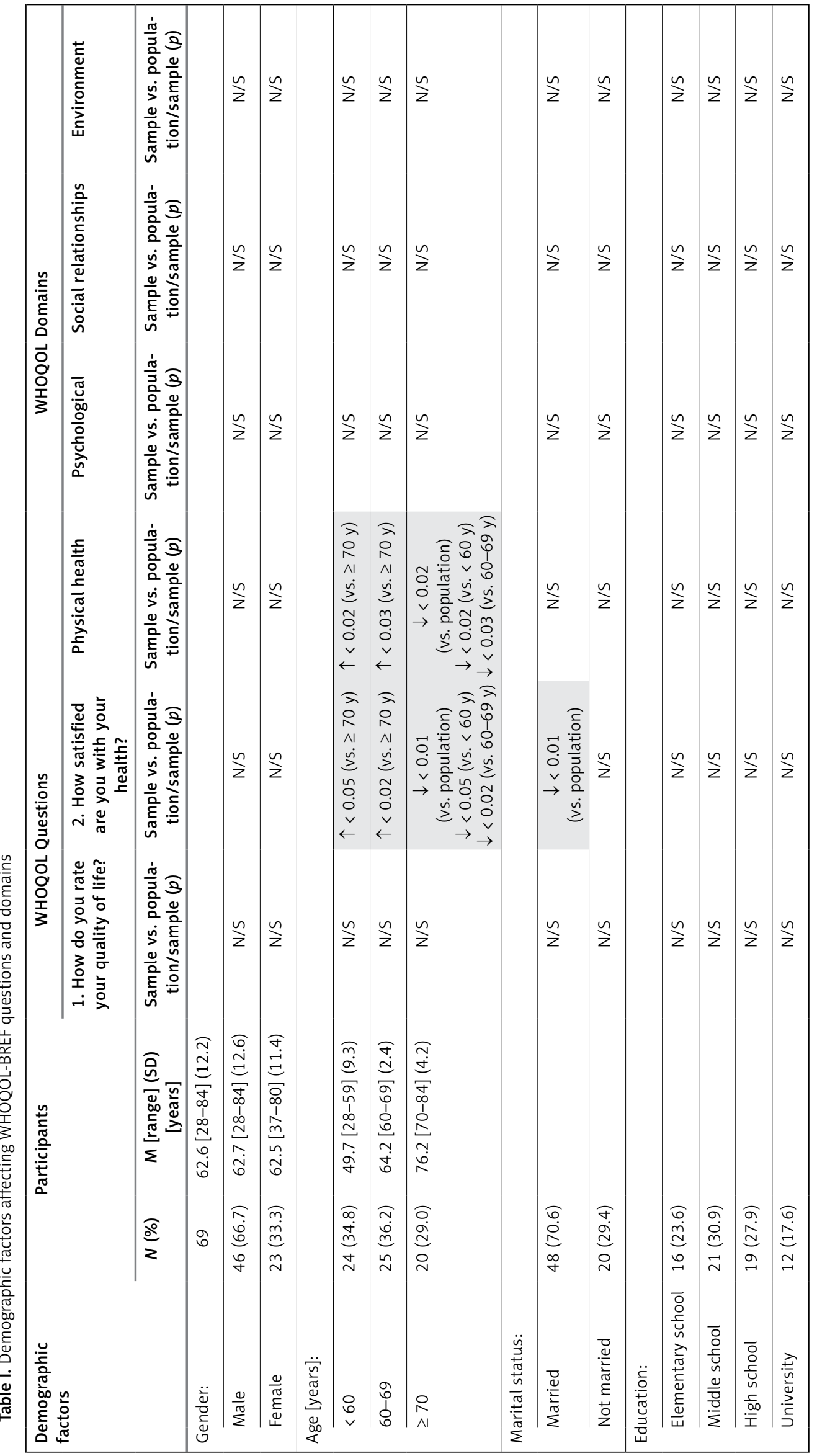




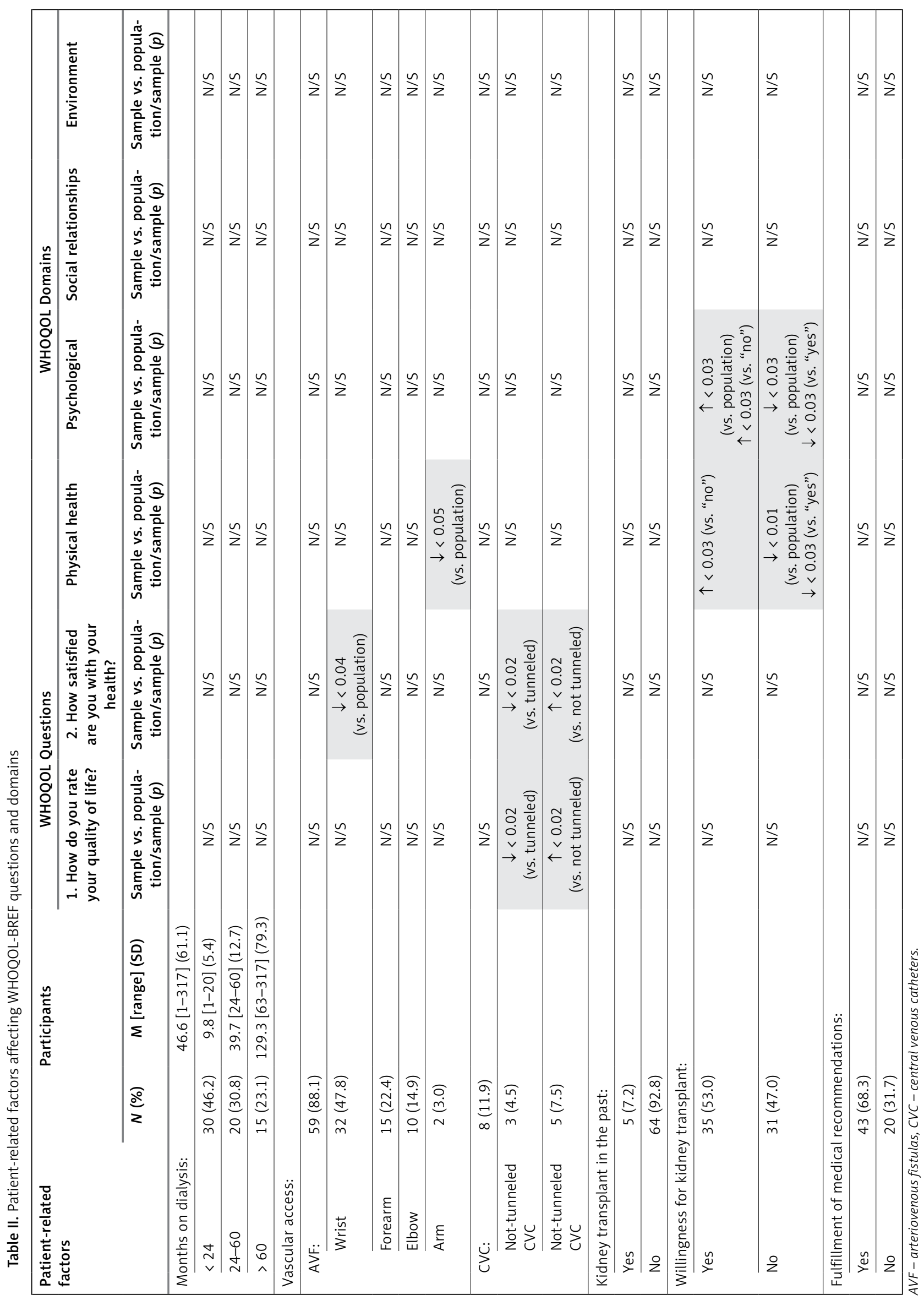




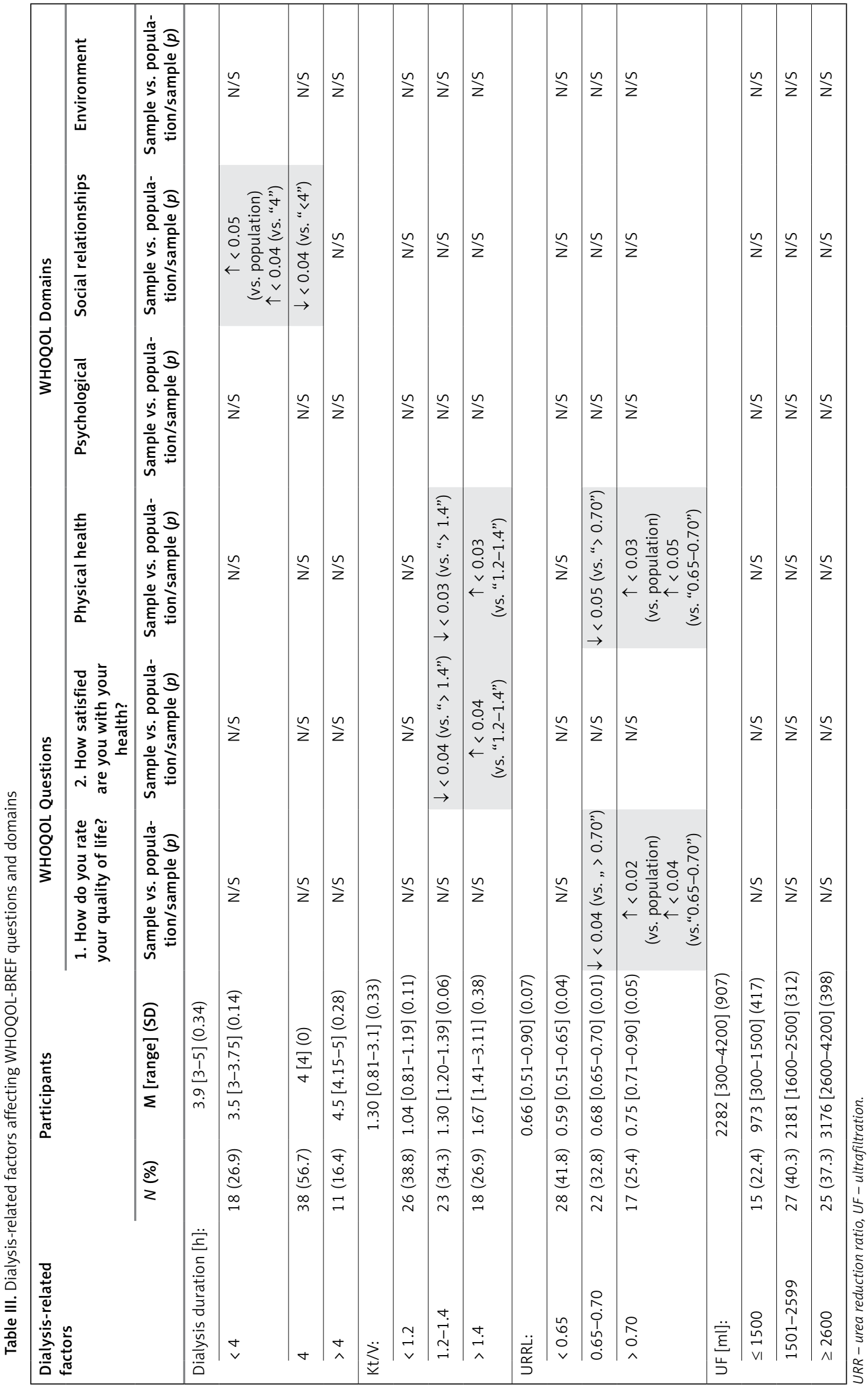




\section{Statistical analysis}

Statistical analysis was performed with StatPac software, version 4.0. Mean, range and standard derivation (SD) were calculated for continuous variables: age, gender, months on $\mathrm{HD}$, dialysis duration, Kt/V, URR and UF. The variables were compared by the Student $t$-test. Means of three or more groups were compared with the ANOVA test. A $p$-value of less than 0.05 was considered to be statistically significant.

\section{Results}

Participants, including 23 (33.3\%) women and $46(66.7 \%)$ men, ranged in age from 28 to 84 , with a mean age of $62.6 \pm 12.2$ (Table I).

No statistically significant relations were found between any of the WHOQOL-BREF domains or questions and the following demographic and patient-related factors: gender, education, months on HD, kidney graft in the past and fulfillment of medical recommendations (Tables I, II).

Despite a lack of age-related differences in the rating of the first question, in the Psychological, Social relationship and Environment domains, the eldest group (over 70 years old) rated significantly lower both the satisfaction with health $(p<0.01)$ and Physical health domain $(p<0.02)$ (Table I).

Married HD patients rated their satisfaction with health (question 2) significantly lower ( $p<$ 0.01). However, marital status did not reflect the rating of overall $\mathrm{QOL}$ (question 1 ) or any of the four WHOQOL-BREF domains (Table I).

HD patients with wrist arteriovenous fistulas (AVFs) were less satisfied with their health (question 2) ( $p<0.04)$, while those with arm AVFs rated the Physical health domain significantly lower $(p<0.05)$. Among HD patients with central venous catheters (CVCs) those with tunneled CVCs rated both their QOL (question 1) and satisfaction with their health (question 2) higher, with $p<0.02$ and $p<0.02$, respectively (Table II). Other significant differences in answering the questions or rating the domains related to vascular access were excluded. Additionally, there were no significant differences between "AVF" and "CVC" patients (Table II).

Although history of kidney graft did not influence the rating of any WHOQOL-BREF questions or domains, those who do not want to receive a kidney graft in the future rate both the Physical health and the Psychological domains lower, with $p<0.01$ and $p<0.03$, respectively (Table II).

Patients whose HD duration does not exceed $4 \mathrm{~h}$ rated the Social relationship domain significantly higher $(p<0.05)$. Influence of HD duration on two independent questions and Physical health, Psychological or Environment domains was excluded (Table III).
Kt/V higher than 1.4 compared with 1.2-1.4 was associated with higher rating of overall satisfaction with health (question 2) and the Physical health domain ( $p<0.04$ and $p<0.03$, respectively). URR higher than 0.70 was associated with higher rating of both overall QOL (question 1) and the Physical health domain $(p<0.02$ and $p<0.03$, respectively). The HD-related factors including $\mathrm{Kt} / \mathrm{V}$, URR and UF did not influence any other domains or questions (Table III).

\section{Discussion}

A growing group of ESRD patients require adequate management, including adequate HD, in order to prolong their life. However, these patients consider HD as a time-consuming procedure, which needs to be repeated three times a week. Additionally, HD may cause QOL impairments $[1,2,16,17]$. The ESRD patients, regardless of the treatment modality, have to manage many adversities: cognitive impairment, physical symptoms, fluid and dietary restriction, and changed body image $[3,5,6,15,18,19]$. They may have many risk factors of reduced QOL such as diabetes, hypertension, dyslipidemia, hypoxemia, proinflammatory state, as well as fluid, osmolar and uremic toxin variations [6]. Additionally, they feel a significant lack of sufficient control over the whole treatment $[3,6,15]$. These factors affect the whole life of ESRD patients, including their physical and social relations. Patients need to reconsider their personal, social and professional goals when living with a chronic illness such as ESRD and undergoing $\mathrm{HD}[3,15,20]$.

WHOQOL-BREF is a self reported QOL inventory [11] (Tables I-III). It was administered to 69 ESRD patients undergoing HD recruited from one hospital-based dialysis unit. They were asked to answer demographic questions, fill in the medical history data along with 26 questions included in WHOQOL-BREF, taking into account the last 2 weeks. Those who had problems filling out the forms could get help from the staff or their families.

The recovery period after a HD session affects QOL. Just after the HD session patients may feel tired and in need of rest or sleep [21]. For this reason our patients were asked to fill in the questionnaire during the day after HD, when their cognitive functions were optimal.

The first question was focused on rating patients' overall QOL. The patients whose URR exceeded 0.70 rated the QOL significantly higher $(p<0.02)$ (Table III). Additionally, patients with tunneled CVCs rated both the overall QOL (question 1) and satisfaction with health (question 2) significantly higher than those with not-tunneled CVCs ( $p<0.02, p<0.02$, respectively) (Table II). However, no significant difference was observed 
between "CVC" and "AVF" groups (Table II). Patients with an acute vascular access such as not-tunneled CVC may not accept the new chronic disease and a time-consuming HD procedure, spending a long time three times a week in the HD unit and being tired for a long time after the HD session $[3,6,15,21]$. These factors may explain the reduced overall rating of both the QOL and the satisfaction with health (Table II).

The satisfaction with health was rated in the second question significantly lower by the eldest patients aged over 70 years $(p<0.01)$, married $(p<0.01)$ and those with wrist AVF $(p<0.05)$ (Tables I, II). Additionally, the eldest patients rated the Physical health domain significantly lower $(p<$ 0.02) (Table I). For the eldest patients the reduced satisfaction with overall health and the Physical health domain may be associated not only with ESRD and HD per se, but also with their age and age-related impairments and weakness [4-6]. In the case of married patients, ESRD and HD affect not only their own life, but also the life of a spouse and therefore may lower satisfaction with overall health (Table I) [6]. Wrist AVF is obviously the best vascular access. However, anastomosis located on the wrist, the scar and the dilated vein easy for others to see may cause discomfort and embarrassment (Table II) [4, 5].

According to the WHO definition health can be considered in a physical, mental and social dimension [1]. The Physical health domain of the WHOQOL-BREF assesses activities of daily living, dependence on medicinal substances and medical aids, energy and fatigue, mobility, pain and discomfort, work capacity, sleep and rest $[11,12]$. The Physical health domain was rated significantly lower not only by the eldest patients ( $p<$ $0.02)$, but also by those with arm AVFs $(p<0.05)$ and those who do not want to receive a kidney graft $(p<0.01)$ (Tables I, II). Patients who do not want to be a recipient of a kidney graft rated both Physical health $(p<0.01)$ and Psychological $(p<$ 0.03) domains lower (Table II). The Physical health domain was rated significantly higher by those with URR over $0.70(p<0.03)$ (Table III). The AVF location at the arm level may follow more distal previous AVFs which failed or be a consequence of peripheral vascular difficulties caused by e.g. diabetes [4, 5]. This may affect many aspects of the patient's life, including the physical dimension $[6,16]$. Unwillingness to be a recipient of a kidney graft, which is the best kidney replacement therapy, may be a result of a lack of energy and mobility necessary to go through the graft procedure as well as a desire to avoid greater dependence on medical staff and medical aids following the graft $[3,15]$. The HD patients besides the dialysis per se need to manage the physical symptoms and dietary regimes and accept changes in the overall body image. They have a higher rate of suicide and more depressive symptoms $[3,22]$. Depression may be caused by HD modality, when patients have to be continually connected to the HD machine and experience significant restrictions in independent living [3]. The HD patients report insufficient availability of health services, difficulties in transportation, finances and opportunities for recreation, and acquiring skills and knowledge [15]. Authors have drawn attention to psychosocial problems of HD patients including the influence of HD on their careers, visits three times a week to the dialysis center and a long time spent on $\operatorname{HD}[3,23]$.

The Psychological domain covers: bodily image and appearance, negative and positive feelings, self-esteem, spirituality, religion and personal beliefs, thinking, learning, memory and concentration $[8,11,12]$. This domain was rated significantly lower by those who do not want to receive a kidney graft $(p<0.03)$, while those who want a kidney graft rated this domain significantly higher $(p<0.03)$ (Table II). Patients who are not satisfied with their body image experience more negative feelings. This may result in unwillingness to receive a transplant and go through a variety of intense post-procedure treatments [3, 8].

The Social relationship domain assesses: personal relationships, social support and sexual activity $[11,12]$. Patients whose HD took less than $4 \mathrm{~h}$ rated the Social relationship domain significantly higher $(p<0.05)$ (Table III). Duration of a single HD is the only factor significantly influencing the Social relationship domain. Authors point out that long HD impedes social relationships. HD patients report experiencing less support from their community. It may be associated with the restrictions on their lives and being dependent on the HD procedure, which may cause social isolation $[15,24]$. The HD treatment is a continuous and stressful condition. The treatment is received in the centre three times a week and the waiting time in the HD unit is relatively long $[3,15]$. When the HD is prolonged, patients spend a longer time in the HD unit three times a week. This may affect patients' careers and cause psychosocial problems. For this reason it is noted that patients may feel that the social support is not sufficient enough [3, 17, 24].

The Environment domain covers: financial resources; freedom, physical safety and security; accessibility and quality of health care and social care; home environment; opportunities for acquiring new information and skills; opportunities for recreation, leisure activities; physical environment such as pollution, noise, traffic, climate, transport $[11,12]$. The demographic, patient-related and di- 
alysis-related factors had no significant impact on the Environment domain scoring (Tables I-III).

The study has some obvious limitations. The first one is the limited population of a single HD unit. The population of ESRD patients is getting old. Also the percentage of coexisting diseases including diabetes is high. This could cause difficulty in completing the questionnaires and may explain why $49.9 \%$ of patients did not participate in the survey. Patients completed the questionnaires at their homes to limit the influence of the researcher.

In conclusion, the factors lowering the scores for particular questions and domains of the WHOQOL-BREF questionnaire were: senility, marriage, wrist and arm AVF, not-tunneled CVC (vs. tunneled), and unwillingness to have a kidney graft.

The factors that increased the scores for particular questions and domains of the WHOQOL-BREF questionnaire were: short HD, tunneled CVC (vs. not-tunneled), and higher URR.

This is a cross-sectional study of the population of a single hospital-based HD unit and the results cannot be generalized. However, the data suggest that the group of HD patients, especially the eldest of them, should be given special attention. It indicates that despite an awareness and a necessity of a kidney graft as the best kidney replacement therapy, there is a large group among the HD patients who do not want to receive this treatment. The medical professionals in HD units should remember that patients may not feel comfortable with their disease and satisfied with their body image affected by disease and therapy and may not accept even a treatment which is recommended.

\section{Acknowledgments}

We would like to thank the World Health Organization for consent for our project and for providing the WHOQOL-BREF instrument and a Code book.

\section{Conflict of interest}

The authors declare no conflict of interest.

\section{References}

1. Yang SC, Kuo PW, Wang JD, Lin MI, Su S. Development and psychometric properties of the dialysis module of the WHOQOL-BREF Taiwan version. J Formos Med Assoc 2006; 105: 299-309.

2. Avramovic M, Stefanovic V. Health-related quality of life in different stages of renal failure. Artif Organs 2012; 36: 581-9.

3. Ginieri-Coccossis M, Theofilou P, Synodinou C, Tomaras V, Soldatos C. Quality of life, mental health and health beliefs in haemodialysis and peritoneal dialysis patients: investigating differences in early and later years of current treatment. BMC Nephrol 2008; 9: 14.
4. Lim HJ, Koo TY, Lee J, et al.; KNOW-KT Study Group. Health-related quality of life of kidney transplantation patients: results from the KoreaN Cohort Study for Outcome in Patients With Kidney Transplantation (KNOW-KT) Study. Transplant Proc 2016; 48: 844-7.

5. Sathvik BS, Parthasarathi G, Narahari MG, Gurudev KC. An assessment of the quality of life in hemodialysis patients using the WHOQOL-BREF questionnaire. Indian J Nephrol 2008; 18: 141-9.

6. O'Lone E, Connors M, Masson P, et al. Cognition in people with end-stage kidney disease treated with hemodialysis: a systematic review and meta-analysis. Am J Kidney Dis 2016; 67: 925-35.

7. Chen JB, Lam KK, Su YJ, et al. Relationship between Kt/V urea-based dialysis adequacy and nutritional status and their effect on the components of the quality of life in incident peritoneal dialysis patients. BMC Nephrol 2012; 13: 39.

8. Soponaru C, Bojian A, lorga M. Stress, coping mechanisms and quality of life in hemodialysis patients. Arch Med Sci Civil Dis 2016; 1: 16-23.

9. Sulkowski L, Matyja M, Walocha JA, Pasternak A. Satisfaction with life among dialyzed patients: a cantril ladder survey. MGM J Med Sci 2018; 5: 6-11.

10. Szabo S. The World Health Organization Quality of Life (WHOQOL) assessment instrument. Spiker B (Ed.), Quality of Life and Pharmacoeconomics in Clinical Trials, Lippincott-Raven, Philadelphia 1996; 355-62.

11. The WHOQOL Group. The World Health Organization Quality of Life Assessment (WHOQOL): development and general psychometric properties. Soc Sci Med 1998; 46: 1569-85.

12. The WHOQOL Group. Development of the World Health Organization WHOQOL-BREF assessment. Psychol Med 1998; 28: 551-8.

13. World Health Organization. Text of the Constitution of the World Health Organization. Office Records of the WHO, 2, WHO, Geneva 1948; 100-9.

14. WHOQOL-BREF. Programme on mental health, World Health Organization, Geneva.

15. Theofilou P. Quality of life in patients undergoing hemodialysis or peritoneal dialysis treatment. J Clin Med Res 2011; 3: 132-8.

16. Katayama A, Miyatake N, Nishi H, et al. Evaluation of physical activity and its relationship to health-related quality of life in patients on chronic hemodialysis. Environ Health Prev Med 2014; 19: 220-5.

17. Coelho-Marques FZ, Wagner MB, Poli de Figueiredo CE, d'Avila DO. Quality of life and sexuality in chronic dialysis female patients. Int J Impot Res 2006; 18: 539-43.

18. Sulkowski L, Matyja M, Pasternak A. Salvage technique for complicated hemodialysis patients with central venous occlusion. J Postgrad Med Edu Res 2018; 52: 152-4.

19. Sulkowski L, Matyja M, Pasternak A. Lipectomy technique as a second-stage procedure for primarily matured, deep outflow vein in obese individuals. Indian J Nephrol 2018; 28: 320-2.

20. Tong A, Wong G, McTaggart S, et al. Quality of life of young adults and adolescents with chronic kidney disease. J Pediatr 2013; 163: 1179-85.

21. Rayner HC, Zepel L, Fuller DS, et al. Recovery time, quality of life, and mortality in hemodialysis patients: the Dialysis Outcomes and Practice Patterns Study (DOPPS). Am J Kidney Dis 2014; 64: 86-94.

22. Siriopol I, Siriopol D, Voroneanu L, Covic A. Predictive abilities of baseline measurements of fluid overload, assessed by bioimpedance spectroscopy and serum $\mathrm{N}$-ter- 
minal pro-B-type natriuretic peptide, for mortality in hemodialysis patients. Arch Med Sci 2017; 13: 1121-9.

23. Mostafa F, Sad I, Elshamaa M, et al. Left ventricular dysfunction by conventional and tissue Doppler echocardiography in pediatric hemodialysis patients: relation with plasma brain natriuretic peptide levels. Arch Med Sci Atheroscler Dis 2018; 3: 18-28.

24. Chiang CK, Peng YS, Chiang SS, et al. Health-related quality of life of hemodialysis patients in Taiwan: a multicenter study. Blood Purif 2004; 22: 490-8. 